\title{
TEMPORO-SPECTRAL COHERENT STRUCTURE OF TURBULENCE AND PRESSURE USING FOURIER AND WAVELET TRANSFORMS
}

\author{
Thai-Hoa Le ${ }^{*}$ \\ Department of Civil and Earth Resources Engineering, Kyoto University, Kyoto- \\ Daigaku-Katsura Campus, Nishikyo-ku, Kyoto 611-8540, Japan
}

Dong-Anh Nguyen

Institute of Mechanics, Vietnamese Academy of Science and Technology, 264 Doican Str., Dongda Dist., Hanoi, Vietnam

Received 16 April 2007

\begin{abstract}
Studying the spatial distribution in coherent fields such as turbulent and turbulent-induced force ones is important to model and evaluate turbulent-induced forces and response of structures on the turbulent flows. Turbulent field-based coherent function is commonly used for the spatial distribution characteristic of induced forces in the frequency domain. This paper will focus to study spectral coherent structure of turbulence and forces in not only the frequency domain using conventional Fourier transform-based coherence, but also temporo-spectral coherent one in the time-frequency plane thanks to wavelet transform-based coherence for more understanding of the turbulence and force coherences and their spatial distributions. Effects of spanwise separations, bluff body flow and flow conditions on coherent structures of turbulence and induced pressure, comparison between turbulence and pressure coherences as well as intermittency of coherent structure in the time-frequency plane will be investigated here.
\end{abstract}

\section{INTRODUCTION}

The gust response prediction of structures or long-span bridges immersed in atmospheric turbulent flows subjected by turbulent-induced forces (or buffeting forces) has been based on a corrected strip theory, by which the spatial distribution characteristic and correlation of turbulent-induced forces on structures must be taken into account. For the sake of simplification, however, the turbulent field can represent for the induced force field. Thus, it is assumed that the spanwise coherence of the buffeting forces is similar to that of the ongoing turbulence (the fluctuating velocity components) that was simplified as an exponential coherent formula in the gust response analysis [4]. Recent literatures, however, found out that the coherence of the buffeting forces was larger than that of the ongoing turbulence [6, $8-10]$. This suggests that influence of structure on the ongoing turbulent flow must not be negligible, and interaction phenomena between ongoing flow and structure might have involved to modification of the ongoing turbulent flow around the structure (one is mentioned as a bluff body flow).

\footnotetext{
* Corresponding author e-mail: thle@bwku.mbox.media.kyoto-u.ac.jp
} 
Uncertainty from force coherence higher than turbulent coherence can cause either underestimation or overestimation on the gust response prediction of structures. Mechanism of higher force coherence, coherent structures of turbulence and induced forces as well as effect of bluff body flow on coherence should be further clarified in order to reduce the analytical uncertainty. Coherent structure of the buffeting forces has been studied ideally by the mean of surface pressure measurement by which the induced forces can be deducted by integration of the surface pressure field around structural cross section. Identification of bluff body flow around structure (such as separation bubble, flow reattachment, vortex shedding), furthermore, can be roughly obtained thanks to chordwise distribution of mean and fluctuating pressures which has been verified by means of smoke visualization $[2,5]$.

The Fourier transform has been most popularly and conventionally used to study in spectralbased computations, physical data analysis, coherent structures in the frequency domain so far [1]. No time information, however, can be obtained from the Fourier transform-based tools such as Fourier coefficient, auto power spectrum, cross power spectrum, coherence and phase difference which have been applied to identify the dominant frequency components and the cross correlation between two given time series in the frequency domain. These tools, moreover, is accurately applicable only for purely stationary time series. Wavelet transform has been recently proposed to represent any time series in a time-scale plane, known as a time-frequency analysis [3]. First-order wavelet coefficient has been used almost so far, however, some wavelet transform-based advanced tools corresponding to conventional Fourier transform-based ones such as wavelet power spectrum, wavelet coherence can be developed to express and detect auto, cross correlations of any time series and between two time series in the time-scale plane $[7,11]$. The wavelet transform-based tools, furthermore, are advantageous over the Fourier transform as a powerfully analyzing tool for non-stationary, non-linear and intermittent time series.

In this paper, the temporal-spectral coherent structures of wind and pressure will be studied using both Fourier transform-based coherence and wavelet transform-based one (for brevity, the Fourier coherence and wavelet coherence). Effects of spanwise separations, bluff body flow and turbulent flow conditions on coherent structures of turbulence and pressure, comparison between wind and pressure coherence as well as intermittency of wavelet coherence are going to be discussed. Physical measurements of the surface pressure and turbulence have been carried out on $2 \mathrm{D}$ rectangular section with side ratio $\mathrm{B} / \mathrm{D}=5$ under artificial turbulent flows in the wind tunnel.

\section{FOURIER TRANSFORM-BASED AND WAVELET TRANSFORM-BASED COHERENCES}

\subsection{Fourier coherence}

Fourier transform can be expressed by Wiener-Kintchine conversion pair between time series $X(t)$ and its spectral representation $\hat{X}(n)$ using the harmonic function as follows:

$$
\hat{X}(n)=\int_{-\infty}^{\infty} X(t) \exp (-j 2 \pi n t) d t ; \quad X(t)=\int_{-\infty}^{\infty} \hat{X}(n) \exp (j 2 \pi n t) d t
$$

where $X(t), \hat{X}(n)$ : time series and its Fourier coefficient; n: Fourier frequency variable.

The Fourier coherence is approximately expressed as the normalized correlation coefficient of two spectral quantities of $X(t)$ and $Y(t)$ in the frequency domain [1]: 


$$
\operatorname{COH}_{X Y}^{2}(n)=\frac{\left|S_{X Y}(n)\right|}{\sqrt{S_{X}(n) S_{Y}(n)}}
$$

where $\mid$ : absolute operator; $S_{X}(n), S_{Y}(n), S_{X Y}(n)$ : Fourier auto power spectra and Fourier cross power spectrum at/between two separated points, respectively defined as:

$$
S_{X}(n)=E\left[\hat{X}(n) \hat{X}(n)^{* T}\right] ; S_{Y}(n)=E\left[\hat{Y}(n) \hat{Y}(n)^{* T}\right] ; S_{X Y}(n)=E\left[\hat{X}(n) \hat{Y}(n)^{* T}\right]
$$

where $\mathrm{E}[]$ : expectation operator; ${ }^{*}, \mathrm{~T}$ : complex conjugate and transpose operators.

\subsection{Wavelet coherence}

The wavelet transform (also called as continuous wavelet transform) of the given time series $X(t)$ is defined as the convolution operator between $X(t)$ and the wavelet function $\psi_{\tau, s}(t)$ :

$$
W_{X}^{\psi}(\tau, s)=\left\langle X, \psi_{\tau, s}\right\rangle=\int_{-\infty}^{\infty} X(t) \psi_{\tau, s}^{*}(t) d t
$$

where $W_{X}^{\psi}(s, \tau)$ : the wavelet coefficients at translation $\tau$ and scale $\mathrm{s}$ in the time-scale plane; the brackets $\langle$,$\rangle denote the convolution operator; \psi_{\tau, s}(t)$ : wavelet function at translation $\tau$ and scale $\mathrm{s}$ of the basic wavelet function $\psi(t)$ (the mother wavelet):

$$
\psi_{\tau, s}(t)=\frac{1}{\sqrt{s}} \psi\left(\frac{t-\tau}{s}\right)
$$

The wavelet coefficients $W_{X}^{\psi}(s, \tau)$ can be considered as a correlation coefficient and a measure of similitude between wavelet and given time series in the time-scale plane. The wavelet scale has its meaning as an inverse of the Fourier frequency, thus inter-relationship between the wavelet scale and the Fourier frequency can be obtained. One would like to develop wavelet transform-based tools such as the wavelet auto spectra and the wavelet cross spectrum at time shift index $i$ and scale s of two signals $X(t)$ and $Y(t)$, based on their wavelet coefficients $W_{X i}(s)$, $W_{Y i}(s)$ can be defined as following formulae:

$$
\begin{gathered}
W P S_{X X i}(s)=\left\langle W_{X i}(s) W_{X i}^{* T}(s)\right\rangle ; W P S_{Y Y i}(s)=\left\langle W_{Y i}(s) W_{Y Y i}^{* T}(s)\right\rangle \\
W C S_{X Y i}(s)=\left\langle W_{X i}(s) W_{Y i}^{* T}(s)\right\rangle
\end{gathered}
$$

where $\operatorname{WPS}_{X X i}(s), W P S_{Y Y i}(s)$ : wavelet auto spectra of $X(t), Y(t) ; W_{C S_{Y Y i}}(s)$ : wavelet cross spectrum between $X(t)$ and $Y(t) ;\langle\rangle$ denotes smoothing operator in both time and scale directions.

With respect to the Fourier coherence, the squared wavelet coherence of $X(t), Y(t)$ is defined as the absolute value squared of the smoothed wavelet cross spectrum, normalized by the smoothed wavelet auto spectra (Torrence and Compo, 1998):

$$
W C O_{X Y i}{ }^{2}(s)=\frac{\left|\left\langle s^{-1} W C S_{X Y i}(s)\right\rangle\right|^{2}}{\left\langle s^{-1}\left|W P S_{X X i}(s)\right|\right\rangle\left\langle s^{-1}\left|W P S_{Y Y i}(s)\right|\right\rangle}
$$


where ||denotes the absolute operator; $\mathrm{s}^{-1}$ is used to convert to an energy density. Similar to Fourier coherence, $0 \leq W C O_{X Y i}{ }^{2}(s) \leq 1$ in which wavelet coherence come to unit, two time series $X(t), Y(t)$ are full-correlated, wavelet coherence to zero, two time series are noncorrelated.

\subsection{Complex Morlet wavelet}

The complex Morlet wavelet is the most applicable for physical measurement analysis in the wavelet transform, thanks to its containing of harmonic components and its analogs to the Fourier transform (see Figure 1):

$$
\begin{gathered}
\psi(t)=(2 \pi)^{-1 / 2} \exp \left(i 2 \pi n_{0} t\right) \exp \left(-t^{2} / 2\right) \\
\hat{\psi}(s n)=(2 \pi)^{-1 / 2} \exp \left(2 \pi^{2}\left(s n-n_{0}\right)^{2}\right)
\end{gathered}
$$

where $n_{0}$, s: central frequency and wavelet scale of the complex Morlet wavelet.
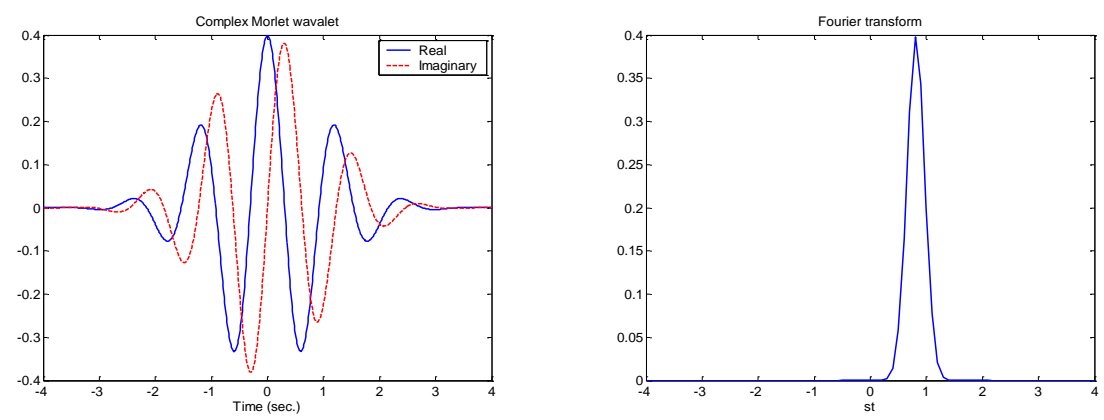

Fig. 1: Complex Morlet wavelet and its Fourier transform.

Inter-relation between the central frequency, wavelet scale and the Fourier frequency can be approximated as following formula:

$$
n=\frac{n_{0} n_{s}}{s}
$$

where $n_{s}$ : sampling frequency of given time series.

\subsection{Time-scale smoothing and end-effect elimination}

Averaging in both time and scale directions must be required in the wavelet transform, especially in computing the wavelet spectrum and wavelet coherence. The averaging techniques of the wavelet spectrum in time and scale at the time-shifted index $\mathrm{p}$ can be expressed by follows (Torrence and Compo, 1998):

Time smoothing:

$$
\left\langle\operatorname{WPS}_{i}^{2}(s)\right\rangle=1 / N_{a} \sum_{i=i_{1}}^{i_{2}}\left|W P S_{i}(s)\right|^{2}
$$


Scale smoothing: $\quad\left\langle W P S_{i}^{2}(s)\right\rangle=\delta j \delta t / C_{\delta} \sum_{j=j_{1}}^{j_{2}}\left|W P S_{i}\left(s_{j}\right)\right|^{2} / s_{j}$

where $\mathrm{j}$ assigned between $\mathrm{j}_{1}$ and $\mathrm{j}_{2} ; \mathrm{N}_{\mathrm{a}}$ : number of averaged points $\left(N_{a}=j_{2}-j_{1}+1\right) ; \delta \mathrm{j}, \delta t$ : factor of window width and sampling period ; $\mathrm{C}_{\delta}$ : constant (complex Morlet wavelet: $\left.\delta \mathrm{j}=0.6, C_{\delta}=0.776\right)$.

Because the wavelet transform deals with finite-length of time series, errors and bias values usually occur at two ends of time series, known as the end effect. One sample solution to eliminate the end effect is to truncate number of discrete results at two ends of time series after the wavelet transform is completed. Removed number, however, depend on the wavelet scale, thus so-called cone of influence should be estimated for more accuracy.

\section{EXPERIMENTAL APPARATUS}

Analyzing data were obtained by physical measurements in the Kyoto University's open-circuit wind tunnel. Motionless testing model was fixed by supports on a working section. The turbulent flow was generated artificially by grid devices which was located in $750 \mathrm{~mm}$ upstream from model's leading edge. Wind turbulence and surface pressures were measured in the three turbulent flows at mean wind velocities $U=3,6$ and $9 \mathrm{~m} / \mathrm{s}$ corresponding to flow case 1 , flow case 2 and flow case 3 , respectively. A rectangular sectional model with side ratio $\mathrm{B} / \mathrm{D}=5$ was rigidly supported in a testing section. Pressure holes were arranged on one side of model surface, consisting of 19 rows in chordwise direction (from position 1 to position 19). Mean and turbulent components (fluctuating velocity components: u-, w-components corresponding to alongwind and crosswind directions, respectively) were measured thanks to a hot-wire anemometer using x-type probes (Model 0252, Kanomax Japan, Inc.) and calibrated and linearized by a constant-temperature anemometer (CTA) (Models 1013, 1011, Kanomax Japan, Inc.). Unsteady surface pressures were measured by multi-channel pressure measurement system (ZOC23, Ohte Giken, Inc.). It is noted that turbulent components and surface pressures were simultaneously obtained in order to investigate in the time domain. Electric signals were filtered by $100 \mathrm{~Hz}$ low-pass filters (E3201, NF Design Block Co., Ltd.) before passed through A/D converter (Thinknet DF3422, Pavec Co., Ltd.) with sampling frequency at $1000 \mathrm{~Hz}$ in 100 seconds.

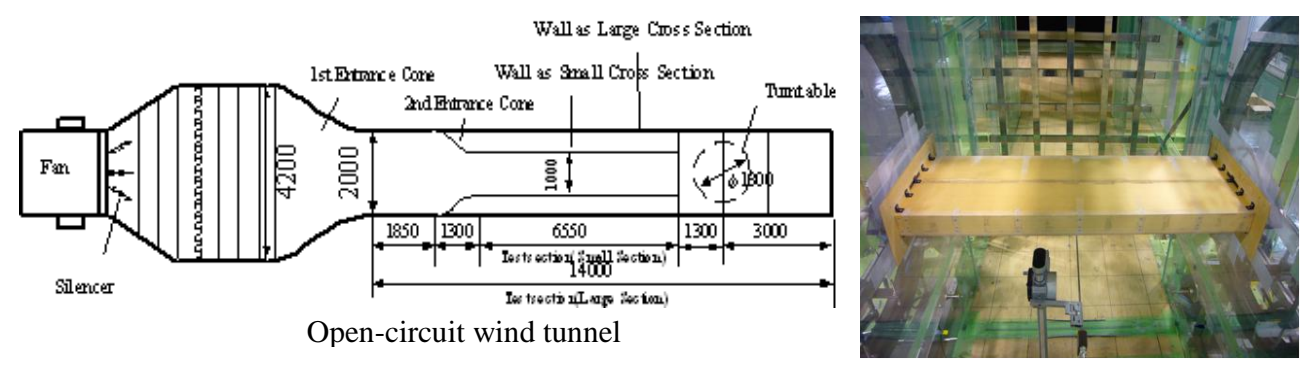

Turbulent flow conditions

\begin{tabular}{|c|c|c|c|}
\hline Flows & Velocity & \multicolumn{2}{|c|}{ Intensity of turbulence } \\
\hline & $\begin{array}{c}\mathrm{U} \\
(\mathrm{m} / \mathrm{s})\end{array}$ & $\begin{array}{c}\mathrm{I}_{\mathrm{u}} \\
(\%)\end{array}$ & $\begin{array}{c}\mathrm{I}_{\mathrm{w}} \\
(\%)\end{array}$ \\
\hline Case 1 & 3 & 11.46 & 11.23 \\
\hline Case 2 & 6 & 10.54 & 9.28 \\
\hline Case 3 & 9 & 9.52 & 6.65 \\
\hline
\end{tabular}

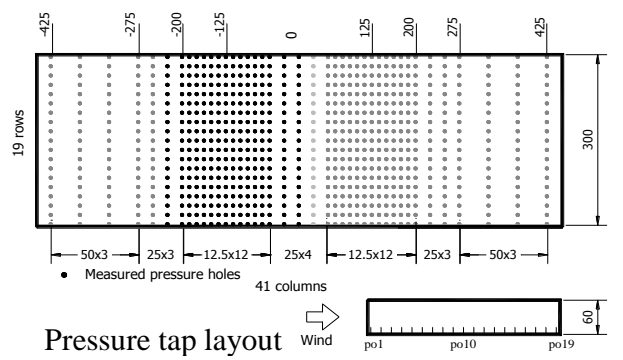

Fig. 2: Wind tunnel configuration, experimental set-ups and pressure tap arrangement. 
Three turbulent flows are characterized by intensities of turbulence corresponding to $\mathrm{u}-\mathrm{w}$ turbulences defined as $I_{u}=\sigma_{u} / \bar{U}, I_{w}=\sigma_{w} / \bar{U}$, where $\sigma_{u}, \sigma_{w}$ : standard deviations of u-, wturbulences, $\bar{U}$ : mean wind velocity. Flow around the model section is identified in the next part.

\section{BLUFF BODY FLOW IDENTIFICATION}

So-called bluff body flow is characterized by flow separation and reattachment as well as formation of vortex shedding on the body surface that are mainly influenced by body geometry, dimension and its motion. Bluff body flow around the body is generated by aerodynamic interaction between the basic ongoing flow and body. Identification of this bluff body flow is usually required for occurrence possibility and oscillatory mechanism of physical phenomena on the model. Experimental flow identification of the bluff body flow is due to flow visualization techniques or due to chordwise pressure distribution on model surface. Mean and root-meansquare fluctuating pressure coefficients have been normalized by dynamic pressure component from unsteady pressure data as follows:

$$
C_{p, \text { mean }}^{(i)}=\bar{p}^{(i)} /\left(0.5 \rho U^{2}\right) ; C_{p, r m s}^{(i)}=\sigma_{p}^{(i)} /\left(0.5 \rho U^{2}\right)
$$

where $\mathrm{i}$ : index of pressure time series; $\bar{p}$ : mean pressure; $\sigma_{p}$ : standard deviation of pressure series.
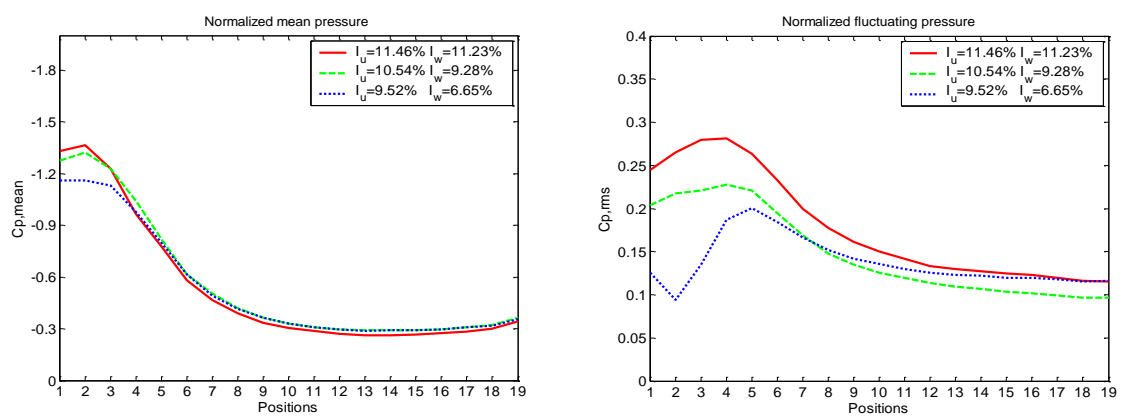

Fig. 3: Normalized mean and fluctuating pressure distributions.

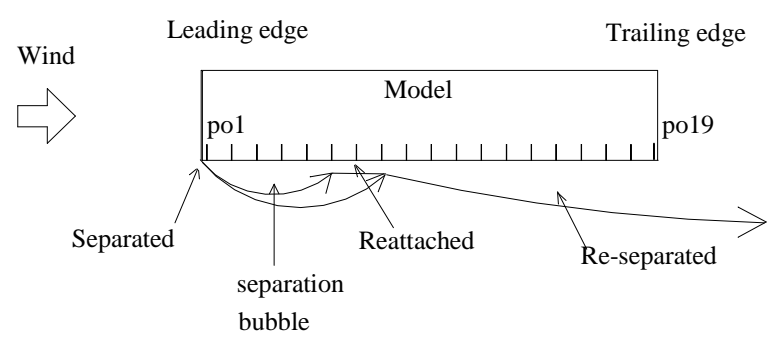

Fig. 4: Bluff body flow pattern. 
Figure 3 shows normalized mean and fluctuating pressure distributions on the chordwise direction from position 1 (at leading edge) to position 19 (at trailing edge). According to Hiller and Cherry (1981), the bluff body flow around model section can be supposed to be separated and reattached flows in which the ongoing turbulent flow separates at sharp corners to build up the separation bubble at leading edge region, then reattach at roughly positions $6,7,8$ (determined near after peaked positions of the fluctuating pressure or at stable value position of the mean pressure) with respect to an increase of mean velocities, at last vortices shed at reattachment point forward the trailing edge (see Figure 4). Thus, length of separation bubble ranges between $\mathrm{X} / \mathrm{D}=1.4 \div 2$ (X: length of separation bubble; $\mathrm{D}$ : model depth). It is roughly predicted that, moreover, positions $2 \div 5$ locate in separation bubble, positions $6 \div 8$ at reattachment points, positions $9 \div 19$ in trailing region.
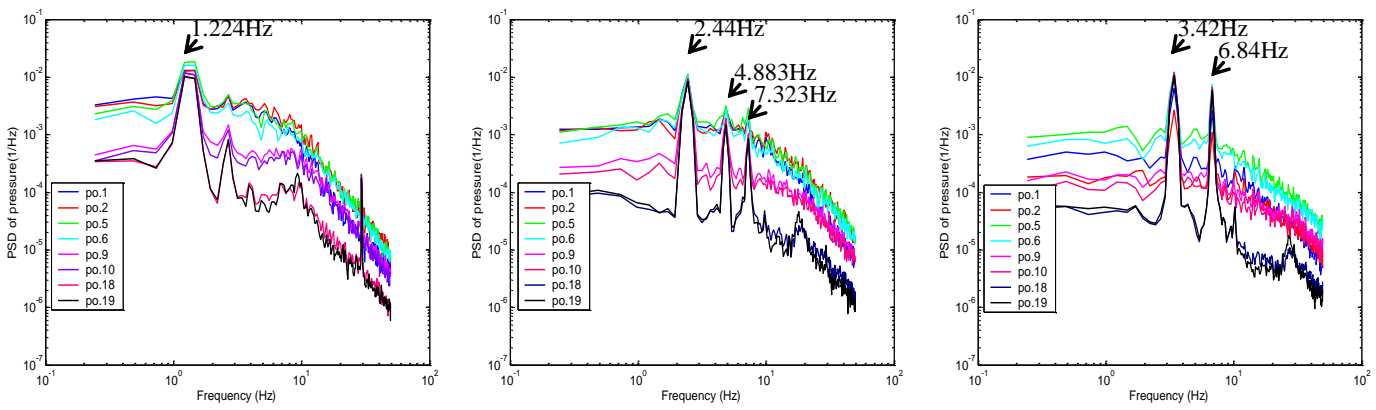

Fig. 5: Auto power spectra (PSD) of normalized fluctuating pressures at turbulent flows: case 1 (left), case 2 (middle) and case 3 (right).

Figure 5 shows auto power spectra (PSD) of the normalized fluctuating pressures in some positions Nos. 1,2,5,6,9,10,18 and 19 at the three turbulent flows. Some frequency peaks are observed at positions in all cases of turbulent flows. Concretely, the frequency peaks have occurred at frequencies $1.224 \mathrm{~Hz}$ and $2.44 \mathrm{H}$ (case 1); at $2.44 \mathrm{~Hz}, 4.883 \mathrm{~Hz}, 4.883 \mathrm{~Hz}$ and $7.324 \mathrm{~Hz}$ (case 2); at $3.42 \mathrm{~Hz}$ and $6.84 \mathrm{~Hz}$ (case 3 ). Moreover, these peaked frequency components that contribute significantly on PSD of fluctuating pressures are observed at some positions Nos. 9, 10, 18 and 19 located at trailing region after reattachment points. It is supposed that the observed frequency peaks are induced by rolled-up vortices shedding away at reattachment points.

\section{RESULTS AND DISCUSSION}

Effects of the spanwise separations, the bluff body flows and turbulent flow conditions on the coherent structures of turbulence and pressure have been investigated thanks to the Fourier coherence. Figure 6 show effect of spanwise separations $(y=25,75,125$ and $225 \mathrm{~mm})$ on the coherences of pressure and turbulence in the frequency band $0 \div 100 \mathrm{~Hz}$. As can be seen that the pressure and turbulent coherences depend much on the spanwise separations on the frequency domain, concretely, coherence reduces with respect to increases of the separations and of observed frequencies. Moreover, effect of the spanwise separation on the pressure coherence seems larger than that on the turbulent coherence in which the turbulent coherences are significant at only the close separation $y=25 \mathrm{~mm}$, but small at the larger separations $\mathrm{y}=75,125$ and $225 \mathrm{~mm}$, whereas pressure coherences are meaning at the spanwise separations $\mathrm{y}=25,75 \mathrm{~mm}$. Coherences of turbulence and pressure are dominated at low frequency band roughly between $0 \mathrm{~Hz}$ and $50 \mathrm{~Hz}$. 

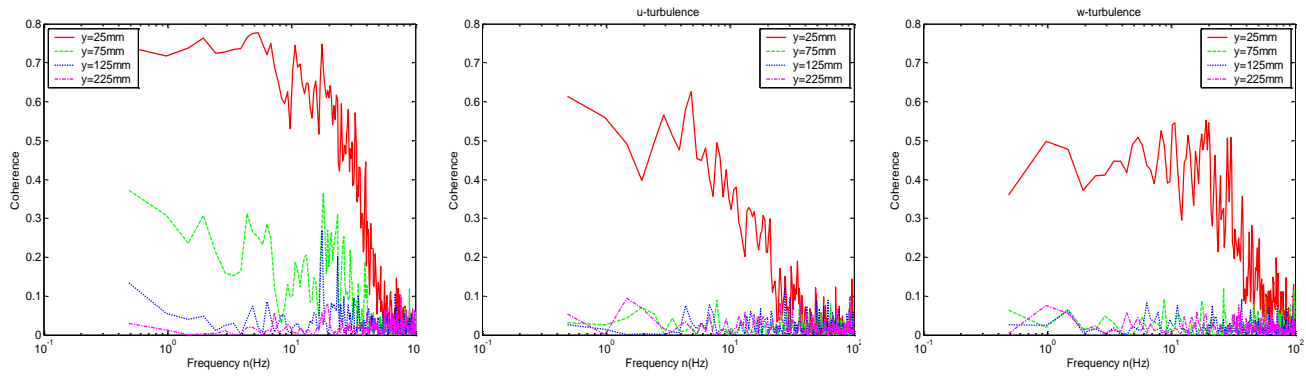

Fig. 6: Effect of spanwise separations on coherences of pressure and turbulence: pressure (left), $u$-turbulence (middle) and w-turbulence (right).
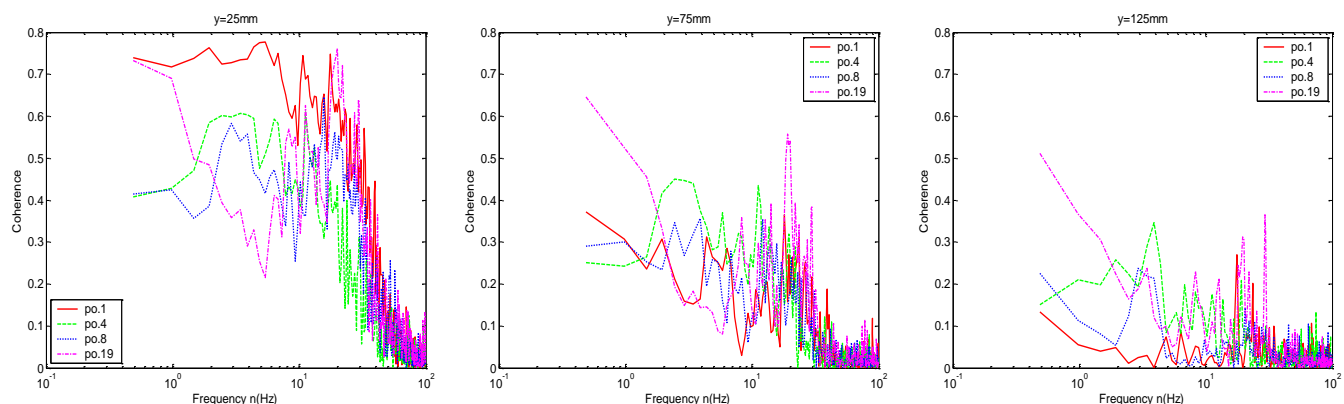

Fig. 7: Effect of bluff body flow on pressure coherences: $y=25 \mathrm{~mm}$ (left), $y=75 \mathrm{~mm}$ (middle) and $y=125 \mathrm{~mm}$ (right).
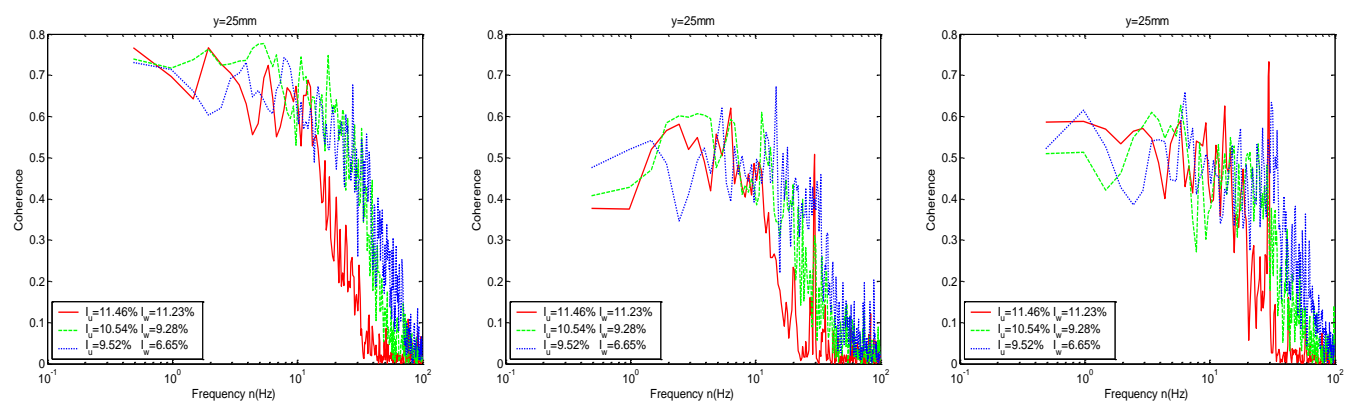

Fig. 8: Effect of turbulent flow conditions of pressure coherences: pressure (left), u-turbulence (middle) and w-turbulence (right).
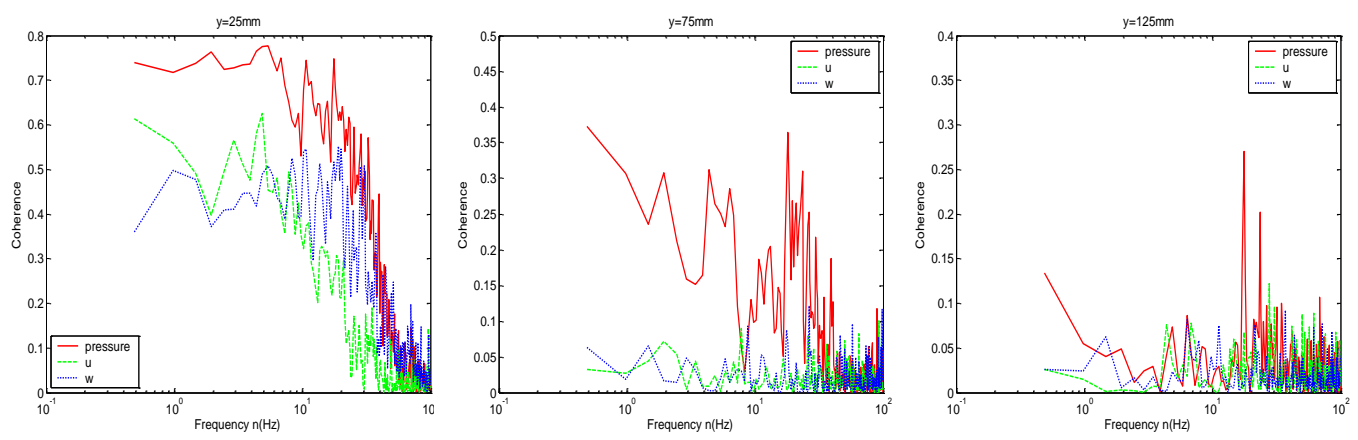

Fig. 9: Comparison between coherences of wind fluctuations and pressure: $y=25 \mathrm{~mm}$ (left), $y$ $=75 \mathrm{~mm}$ (middle) and $y=125 \mathrm{~mm}$ (right). 
Figure 7 expresses the coherences of pressures at some positions Nos. 1, 4, 8 and 19 in the chordwise direction and at the spanwise separations $y=25,75$ and $125 \mathrm{~mm}$. As the bluff body flow is above-identified, the pressure positions Nos.1, 4, 8 and 19 are referred to locate at, in leading edge, separation bubble, reattachment point and trailing edge, respectively. Accordingly, effect of bluff body flow on the coherent structures of turbulence and pressure can be investigated. Concretely, spanwise coherence of pressure at position 1 (at the leading edge) seem to be strong in the close separation $\mathrm{y}=25 \mathrm{~mm}$ and to be small at distant separations $\mathrm{y}=$ $75,125 \mathrm{~mm}$; strong in all separations at position 4 (in the separation bubble); small in all separations at position 8 (at the attachment point); and seem to be small in close separation y = $25 \mathrm{~mm}$ and strong in distant separations $\mathrm{y}=75,125 \mathrm{~mm}$ at position 19 (at the trailing edge) (see Figure 7). Thus, it is supposed that the spanwise coherences seem to be relatively high at positions in the separation bubble region, to be relatively small at positions in the reattachment region. This high coherence at the separation bubble can be explained due to locally-circulated vortices inside the separation bubble as well as rolled-up vortices on the separation bubble surface to enhance spanwise convection of the bluff body flow. Thus, so-called secondary spanwise flow [4] observed at the separation bubble region reasons for the high coherence of pressure. Relatively small coherence observed at the reattachment points might be due to intermittency of the bluff body flow at this region. Reason for changing of the pressure coherences followed spanwise separations at the leading edge and the trailing edge is not clear, maybe sudden contact between basic ongoing flow and model surface as well as vortex shedding away from reattachment region toward the wake might be involved.

Effect of the three flow conditions on the spanwise coherence of pressure and turbulence at the separation $\mathrm{y}=25 \mathrm{~mm}$ is presented in Figure 8. Coherences of pressure and turbulence seem to reduce with respect to increase of intensity of turbulence (or decrease of mean velocities). It can be explained that the low intensity of turbulence (high mean velocity) trends to enhance the spanwise convection and the secondary flow, whereas the high intensity of turbulence (low mean velocity) trends to ruin formation of separation bubble and vortex shedding to resist the spanwise convection of the bluff body flow.

Figure 9 shows comparison between coherence of pressure and that of turbulence at the spanwise separations $y=25,75$ and $125 \mathrm{~mm}$. Obviously, the spanwise coherence of pressure exhibits higher than that of turbulences at the same separation. This finding is good agreement with previous literatures [10]. It is argued that physical interaction between basic ongoing flow and model reasons mechanism of the high coherence of pressure. Coherent structure of turbulence, moreover, exhibits more homogeneously than that of pressure due to interaction of basic flow and model to enhance the spanwise convection and the secondary flow, then to increase coherence of pressure. It is also observed from this Figure that frequency peaks of pressure coherences that characterize for contributions of major frequency components on high coherent events, moreover, do not correspond to those of turbulent coherence. These observations agree well with results and comments of Figure 6 that spatial correlation of the pressure field and induced forces is higher and more complex than that of turbulent field, as well as spectral coherent structures of pressure and turbulence are not compatible together.

Coherent structures of pressure and turbulence have been studied in the time-frequency plane using the wavelet coherence. The wavelet auto spectra (WPS) and wavelet cross spectra (WCS) of pressure and turbulence are calculated following Eq.(6), however, the wavelet cross spectra do not being presented here for sake of brevity. Figure 10 shows the wavelet auto spectra of pressure and turbulence in the frequency band $0 \div 50 \mathrm{~Hz}$ and time interval $0 \div 100$ s $(5$-second intervals in two ends were truncated for the end-effect elimination) at spanwise separation planes $\mathrm{y}=0,25$ and $75 \mathrm{~mm}$. It is observed that the wavelet power spectra of pressure and turbulence distribute intermittently both in the frequency domain and the time domain. Significant events where wavelet power spectra are high, moreover, have occurred discretely at only local areas on the entire time-frequency plane. Wavelet spectra of pressures concentrate 
locally at low frequencies in $0 \div 50 \mathrm{~Hz}$ band, whereas wavelet spectra of turbulence distribute more immensely in this investigated frequency band.

Wavelet coherence (WCO) of pressure and turbulence can be obtained after estimating the wavelet auto and cross spectra. Figure 11 shows the wavelet coherence of pressure and turbulence in $50 \mathrm{~Hz}$ low-pass band and 100-second interval at spanwise separations y $=25,75$, $125 \mathrm{~mm}$ in the time-frequency plane. Some comments can be given from Figure 11. Firstly, similar to the previous results from the Fourier coherence, the wavelet coherence also indicate that coherence reduce with increase of spanwise separations, furthermore, coherence of pressure higher than that of turbulence as well. However, the coherences of pressure and turbulence are represented in the time-frequency plane when coherences are localized and determined at certain time and frequency. Secondly, the coherences of pressure and turbulence are also distributed intermittently in the time-frequency plane. This implies that the intermittent distribution of coherences of pressure and turbulence is observed as the nature of coherence in the time-frequency plane. Thirdly, high coherence events still exist in both coherences of pressure and turbulence at even distant separations but in localized time-frequency areas, this can not clarified from the conventional Fourier coherence where averaging technique in the time domain has been carried out. Finally, the high coherence events of pressure and turbulence do not occur at the same time even time series of pressure and turbulence have been measured simultaneously.
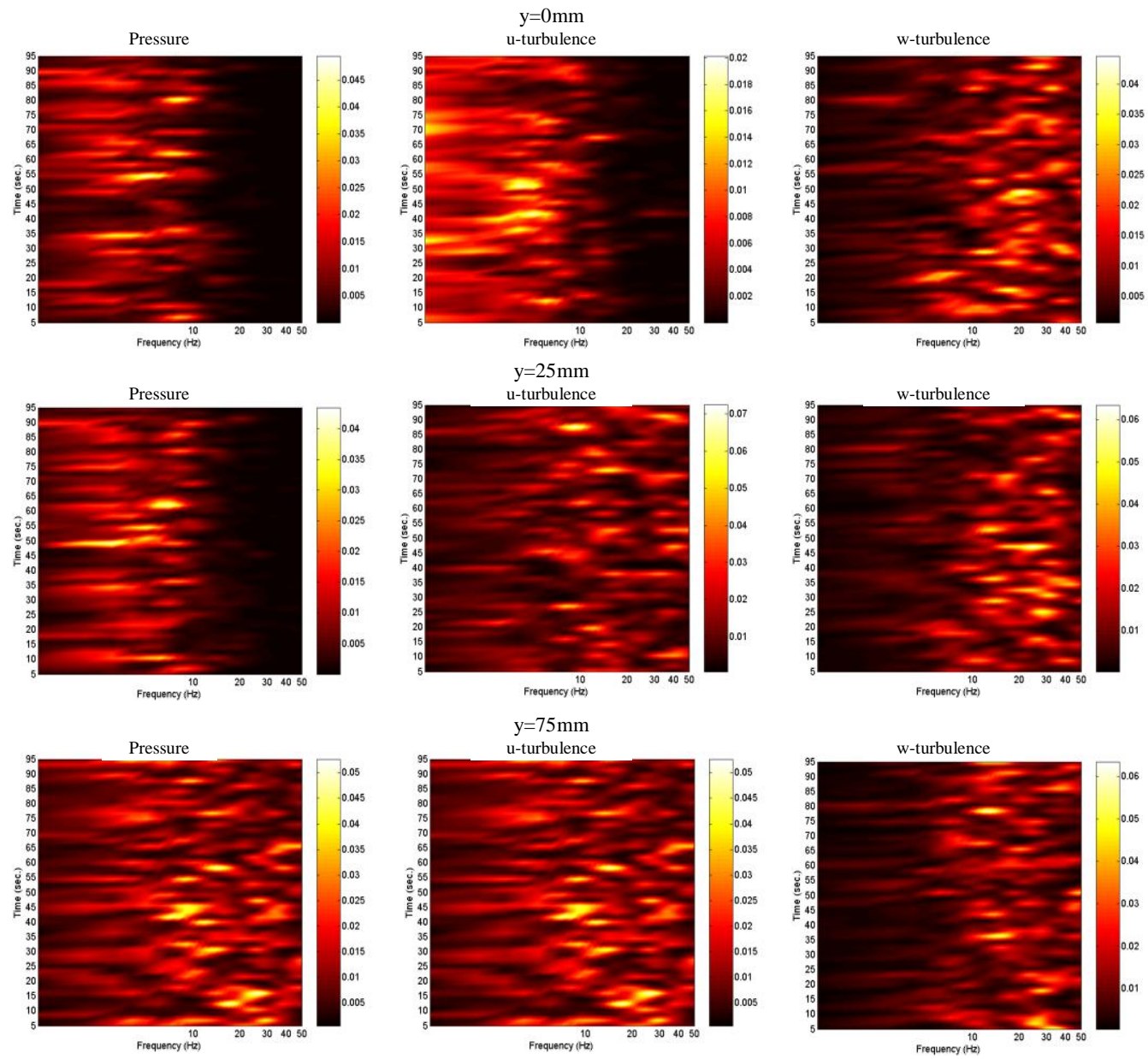

Fig. 10: Wavelet auto spectra (WPS) of pressure and turbulence: $y=0 \mathrm{~mm}$ (upper), $y=25 \mathrm{~mm}$ (middle) and $y=75 \mathrm{~mm}$ (lower). 

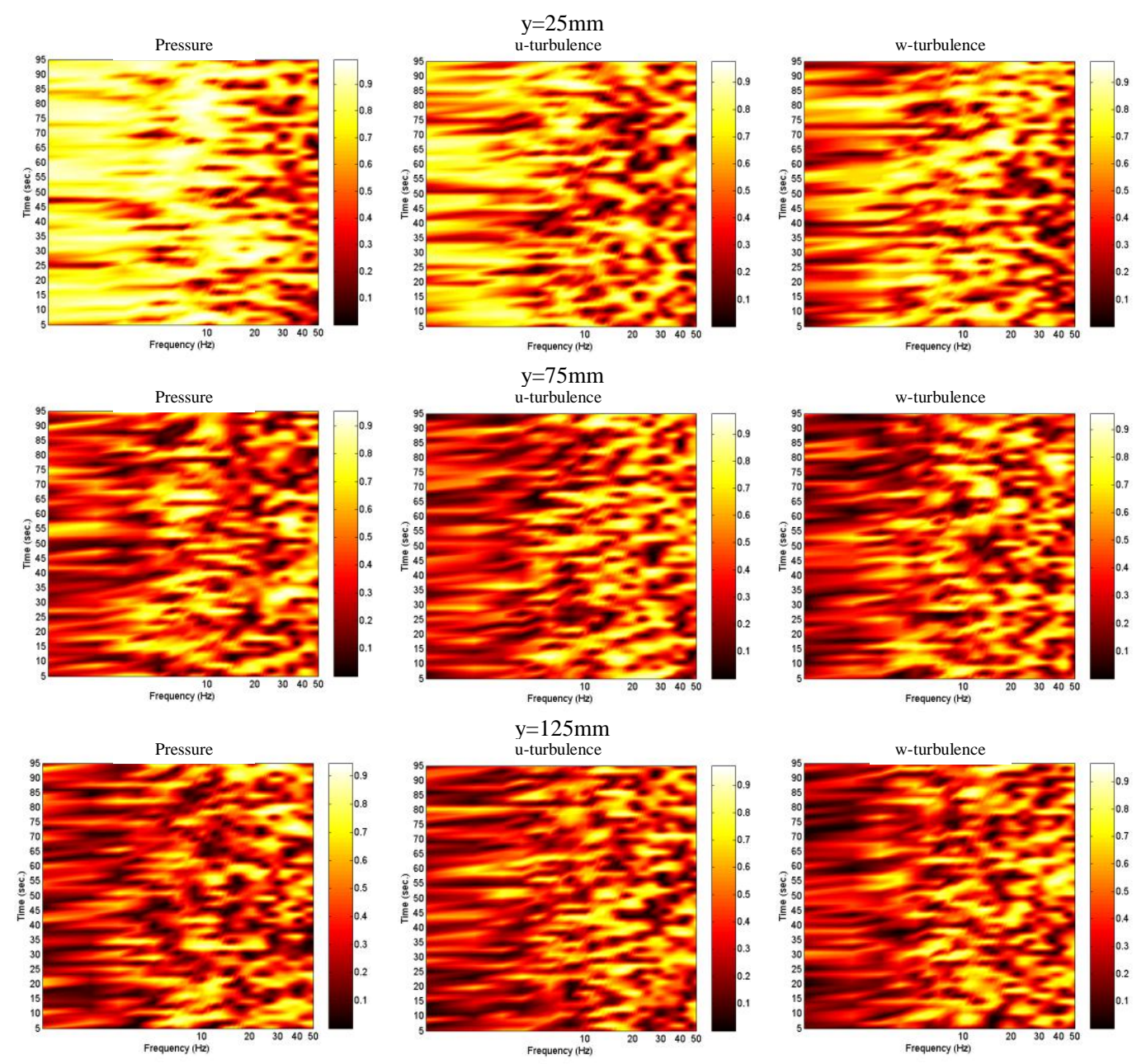

Fig. 11: Wavelet coherence (WCO) of pressure and turbulence: $y=25 \mathrm{~mm}$ (upper), $y=75 \mathrm{~mm}$ (middle) and $y=125 \mathrm{~mm}$ (lower).

From results of the Fourier coherence and wavelet coherence, it is agreed that the coherence of pressure (or forces in general) is higher than coherence of turbulence. Thus, recent model of spatial distribution of turbulent-induced forces based on simplified coherence of turbulence brings obvious uncertainties in modeling force field and predicting response. Furthermore, the high coherence events of pressure do not occur simultaneously corresponding to those of the high coherence ones of wind fluctuations in the time-frequency plane. This expands previous finding from the Fourier coherence that the high coherence events of pressure and turbulence do not simultaneously occur at the same frequency. This also contradicts to the linearized 'quasisteady theory' where simultaneous compatibility in the time domain between turbulence and turbulent-induced pressure/ forces has been assumed.

\section{CONCLUSION}

The temporo-spectral coherent structures of turbulence and surface pressure have been discussed in the frequency domain and the time-frequency plane. As above-discussed, it can be concluded with some following points:

(1) Coherent structures of turbulence and induced pressure depend on some parameters such as the ongoing flow, the spatial separations, the bluff body flow as interactive influence 
between ongoing turbulent flow and structure. This suggests that empirical formula of the coherence function must account for these parameters including the bluff body flow, not only such the ongoing flow, the separation as existing formulae. Moreover, the coherent structure of the turbulence and pressure distribute discretely depending on not only the frequency, but the time.

(2) Coherent structures of turbulence and pressure is represented in the time-frequency plane, by which coherence is significant at the low spectral band and distribute intermittently and locally in the time domain. Thus, intermittency in time and frequency and low-band spectral distribution of the coherent structures of turbulence and pressure can be considered as its nature in the time-spectral plane.

(3) No compatibility and simultaneous occurrence between significant events of high coherence of turbulence and pressure have been observed in the time-frequency plane. Additionally, the spanwise coherence of the surface pressure exhibits larger than that of turbulences at the same separation. These cause some recent uncertainties in the turbulentinduced response prediction of structures relating to the turbulent-induced force model based on the 'quasi-steady theory' and the spatial force correlation using the turbulence coherence.

Further studies should be required for more understanding on coherent structure and spatial correlation of the turbulent-induced forces for refinement of the aerodynamic response prediction of structures.

\section{ACKNOWLEDGEMENTS}

The first author would acknowledge Prof. Matsumoto, Prof. Shirato and Dr. Yagi for their supports this research. The thanks would express to Messrs Yamane, Furukawa and Sumikura for their collaboration in experiments.

\section{REFERENCES}

1. Bendat, J.S. and Piersol, A.G. (2000), Random data: analysis and measurement procedures, 3rd Edition, John Wiley and Sons.

2. Cherry, N.J., Hillier, R., and Latour, M.E.M. (1984), Unsteady measurements in a separated and reattaching flow, Journal of Fluid Mechanics, 144, pp. 13-46.

3. Daubechies, I. (1992), Ten lectures on wavelets, Society for Industrial and Applied Mathematics (SIAM).

4. Davenport, A.G. (1963), The response of slender, line-like structures to a gusty wind, Proceedings of Institution of Civil Engineers, 23, pp. 389-408.

5. Hillier, R. and Cherry, N.J. (1981), The effects of stream turbulence on separation bubbles, Journal of Wind Engineering and Industrial Aerodynamics, 8, pp. 49-58.

6. Jakobsen, J.B. (1997), Span-wise structure of lift and overturning moment on a motionless bridge girder, Journal of Wind Engineering and Industrial Aerodynamics, 69-71, pp. 795805.

7. Kareem, A. and Kijewski, T. (2002), Time-frequency analysis of wind effects on structures, Journal of Wind Engineering and Industrial Aerodynamics, 90, pp. 1435-1452.

8. Kimura, K., Fujino, Y., Nakato, S., and Tamura, H. (1997), Characteristics of buffeting forces on plat cylinders, Journal of Wind Engineering and Industrial Aerodynamics, 69-71, pp. 365-374 
9. Larose, G.L. (1996), The span-wise coherence of wind forces on streamlined bridge decks, Proceedings of the Third International Colloquium on Bluff Body Aerodynamics and Applications, Blacksburg, USA.

10. Matsumoto, M., Shirato, H., Araki, K., Haramura, T., and Hashimoto, T. (2003), Spanwise coherence characteristics of surface pressure field on 2-D bluff bodies, Journal of Wind Engineering and Industrial Aerodynamics, 91, pp. 155-163.

11. Torrence, C. and Compo, G.P. (1998), A practical guide to wavelet analysis, Bulletin of the American Meteorological Society, 79(1), pp. 61-78. 